\title{
Spectral method for the time-dependent Gross-Pitaevskii equation with a harmonic trap
}

\author{
Claude M. Dion and Eric Cancès \\ CERMICS, École Nationale des Ponts et Chaussées, 6 \& 8 avenue Blaise Pascal, Cité Descartes, Champs-sur-Marne, \\ 77455 Marne-la-Vallée, France
}

(Received 5 July 2002; published 15 April 2003)

\begin{abstract}
We study the numerical resolution of the time-dependent Gross-Pitaevskii equation, a nonlinear Schrödinger equation used to simulate the dynamics of Bose-Einstein condensates. Considering condensates trapped in harmonic potentials, we present an efficient algorithm by making use of a spectral-Galerkin method, using a basis set of harmonic-oscillator functions, and the Gauss-Hermite quadrature. We apply this algorithm to the simulation of condensate breathing and scissor modes.
\end{abstract}

DOI: 10.1103/PhysRevE.67.046706

PACS number(s): 02.70.Hm, 03.75.Kk, 31.15.-p

\section{INTRODUCTION}

The experimental realization of Bose-Einstein condensation [1-3] has prompted much work on the study of the dynamics of these condensates. From the theoretical side, many interesting results have been obtained using the GrossPitaevskii equation (GPE) [4-6],

$$
i \hbar \frac{\partial \Psi}{\partial t}=\left[-\frac{\hbar^{2}}{2 m} \nabla^{2}+V_{\mathrm{ext}}+\frac{4 \pi \hbar^{2} a N}{m}|\Psi|^{2}\right] \Psi,
$$

with the normalization condition $\|\Psi(t)\|_{L^{2}}=1 \forall t$, to describe the order parameter $\Psi$ (also called the condensate wave function) of $N$ condensed bosons of mass $m$, interacting via a contact potential described by the scattering length $a$, and eventually confined by an external potential $V_{\text {ext }}$. Even though the Gross-Pitaevskii equation is based on the approximation that all bosons are in the condensed phase ( $T$ $=0 \mathrm{~K})$, direct comparison between theoretical and experimental results have shown that, in many cases, solutions of the GPE contain the essential physics of the underlying phenomena [7-10]. This nonlinear Schrödinger equation (NLSE) has been used, in its time-dependent form, to investigate many aspects of the dynamics of Bose-condensed gas, such as the formation of vortices [11], the interference between condensates [12], of the possibility of creating atom lasers $[13,14]$, to mention only a few.

Most of these and other numerical studies of the timedependent GPE are based on grid methods, i.e., discretize the spatial coordinates on a grid of points, the resulting differential equation being usually solved by Crank-Nicholson or split-operator Fourier methods (see, e.g., Refs. [15-18]). We must point out that, while much care must be taken in solving Eq. (1) because of the nonlinearity, we find, to our dismay, that many authors give results calculated with the timedependent GPE without even specifying what method they have used for their numerical simulation.

In this paper, we wish to focus our attention on the case where the Bose-Einstein condensate is in a (possibly anisotropic) harmonic trap, i.e.,

$$
V_{\mathrm{ext}}(X, Y, Z)=\frac{1}{2} m\left(\omega_{x}^{2} X^{2}+\omega_{y}^{2} Y^{2}+\omega_{z}^{2} Z^{2}\right)
$$

which is the case for most experimental setups $[19,20]$. The method we propose is based on the spectral decomposition of $\Psi$ on a basis of harmonic-oscillator wave functions. In such a representation, the kinetic + trapping potential part of the Hamiltonian is diagonal. The nonlinear part is computed by forward and backward transformations from the spectral to a grid representation. By judicious use of the Gauss-Hermite quadrature, this can lead to an algorithm that is more efficient than those based on grid methods. Although this is akin to discrete variable representation (DVR) methods based on Hermite polynomials, which have been successfully used for the time-independent and time-dependent GPE [21,22], our method is distinct, since our Hamiltonian is expressed in the spectral representation for both the kinetic and potential operators.

We expose in Sec. II our spectral method and the resulting algorithm. We then present different time-evolution schemes that can be used in combination with the spectral method. We finally give in Sec. IV some results that can be obtained from the numerical simulation of the time-dependent GPE, namely, the study of condensate breathing and scissor modes.

\section{SPACE DISCRETIZATION}

To simplify the calculation, we will first rescale Eq. (1) in the three spatial dimensions $(X, Y, Z)$ and in time,

$$
\begin{gathered}
X=\left(\frac{\hbar}{m \omega_{x}}\right)^{1 / 2} x, \\
Y=\left(\frac{\hbar}{m \omega_{y}}\right)^{1 / 2} y, \\
Z=\left(\frac{\hbar}{m \omega_{z}}\right)^{1 / 2} z, \\
t=\frac{1}{\omega_{z}} \tau .
\end{gathered}
$$

We also introduce a new wave function $\psi$ defined as

$$
\Psi(t, X, Y, Z)=A \psi(\tau, x, y, z),
$$

and, considering the normalization condition 


$$
\int_{\mathbb{R}^{3}}|\Psi(t, X, Y, Z)|^{2} d X d Y d Z=1 \forall t
$$

we choose

$$
A=\left(\frac{m}{\hbar}\right)^{3 / 4}\left(\omega_{x} \omega_{y} \omega_{z}\right)^{1 / 4}
$$

such that

$$
\int_{\mathbb{R}^{3}}|\psi(\tau, x, y, z)|^{2} d x d y d z=1 .
$$

The Gross-Pitaevskii equation therefore becomes

$$
\begin{aligned}
i \frac{\partial \psi}{\partial \tau}= & {\left[\frac{\omega_{x}}{\omega_{z}}\left(-\frac{1}{2} \nabla_{x}^{2}+\frac{x^{2}}{2}\right)+\frac{\omega_{y}}{\omega_{z}}\left(-\frac{1}{2} \nabla_{y}^{2}+\frac{y^{2}}{2}\right)\right.} \\
& \left.+\left(-\frac{1}{2} \nabla_{z}^{2}+\frac{z^{2}}{2}\right)+\lambda|\psi|^{2}\right] \psi,
\end{aligned}
$$

with

$$
\lambda=4 \operatorname{\pi aN}\left(\frac{m}{\hbar} \frac{\omega_{x} \omega_{y}}{\omega_{z}}\right)^{1 / 2} .
$$

Coordinate $z$ should be chosen such that $\omega_{z}$ is the greatest of the three frequencies [this is related to the arbitrary choice of the scaling factor in Eq. (3d)].

As all the physical parameters have been absorbed in the nonlinear parameter $\lambda$, calculations with the same $\lambda$ can correspond to results for different species, but in diverse experimental conditions. We can define acceptable lower and upper bounds for $\lambda$ by considering the effective range of the different physical parameters. Considering only cases where the interparticle interaction is repulsive, i.e., $a>0$ and therefore $\lambda>0$, at the lower end we can consider a small ${ }^{4} \mathrm{He}^{*}$ condensate ( $m=4.0$ amu, $a=302$ a.u. [23]) of $N=10^{3}$ atoms in a highly anisotropic $\omega_{x} \omega_{y} / \omega_{z}=2 \pi \times 10^{-1} \mathrm{~Hz}$ trap, giving $\lambda \approx 1.3$, while for a bigger $N \sim 10^{6}$ condensate of heavy atoms such as ${ }^{87} \mathrm{Rb}(m=86.9$ amu, $a=106$ a.u. [24]), $\lambda$ can reach $10^{5}$ for isotropic traps. In the following, we will restrict our study to $\lambda$ in the range $1-10^{3}$, considering that the Thomas-Fermi approximation can be used for greater values of $\lambda[21]$.

\section{A. The spectral-Galerkin method in 1D}

For pedagogical purposes, we first explain our numerical method on the simple case of the one-dimensional (1D) NLSE

$$
i \frac{\partial \psi}{\partial t}(t, x)=H_{0} \psi(t, x)+\lambda|\psi(t, x)|^{2} \psi(t, x),
$$

with

$$
H_{0}=-\frac{1}{2} \frac{\partial^{2}}{\partial x^{2}}+\frac{1}{2} x^{2} .
$$

Extensions to the three-dimensional case will be detailed in the following section.

Denoting by $\psi(t)$ the function $x \mapsto \psi(t, x)$, it can be proven [25] that if

$$
\begin{aligned}
\psi_{0} \in \mathcal{X}:= & \left\{\chi \in L^{2}(\mathbb{R}), \quad \int_{\mathbb{R}}\left|\frac{\partial \chi}{\partial x}\right|^{2}<+\infty,\right. \\
& \left.\int_{\mathbb{R}} x^{2}|\chi(x)|^{2} d x<+\infty\right\},
\end{aligned}
$$

Eq. (6) with initial condition $\psi_{0}$ has a unique solution in $C^{0}\left(\left[0,+\infty[, \mathcal{X}) \cap C^{1}\left(\left[0,+\infty\left[, L^{2}(\mathbb{R})\right)\right.\right.\right.\right.$ and that both the $L^{2}$ norm

$$
\|\psi(t)\|_{L^{2}}=\left[\int_{\mathbb{R}}|\psi(t, x)|^{2} d x\right]^{1 / 2},
$$

and the energy

$$
E=\left(H_{0} \psi(t), \psi(t)\right)+\frac{\lambda}{2} \int_{\mathrm{R}}|\psi(t, x)|^{4} d x
$$

are conserved by the dynamics. A variational formulation of Eq. (6), supplemented by the initial condition $\psi(t=0)$ $=\psi_{0}$, where $\psi_{0} \in \mathcal{X}$ reads

Search $\psi \in C^{0}([0, T], \mathcal{X}) \cap C^{1}\left([0, T], L^{2}(\mathbb{R})\right)$ such that

$$
\forall \chi \in \mathcal{X}, \quad i \frac{d}{d t}(\psi(t), \chi)=\left(H_{0} \psi(t), \chi\right)+\lambda\left(|\psi(t)|^{2} \psi(t), \chi\right),
$$

$$
\psi(0)=\psi_{0}
$$

Numerical solutions can then be obtained by approximating problem (7) with a Galerkin method: a finite-dimensional subspace $\mathcal{X}_{N}$ of the infinite-dimensional vector space $\mathcal{X}$ being given, we consider

Search $\psi_{N} \in C^{1}\left([0, T], \mathcal{X}_{N}\right)$ such that

$$
\forall \chi_{N} \in \mathcal{X}_{N}
$$

$$
i \frac{d}{d t}\left(\psi_{N}(t), \chi_{N}\right)=\left(H_{0} \psi_{N}(t), \chi_{N}\right)+\lambda\left(\left|\psi_{N}(t)\right|^{2} \psi_{N}(t), \chi_{N}\right),
$$

$$
\psi_{N}(0)=\psi_{0}
$$

Denoting by $\left(\phi_{0}, \ldots, \phi_{N}\right)$ an orthonormal basis of $X_{N}$ for the $L^{2}$ scalar product and by $C(t)=\left[c_{n}(t)\right]_{0 \leqslant n \leqslant N}$ the vector of $\mathbb{C}^{N+1}$ collecting the coefficients of $\psi_{N}(t)$ in the basis $\left(\phi_{0}, \ldots, \phi_{N}\right)$, i.e.,

$$
\psi_{N}(t, x)=\sum_{n=0}^{N} c_{n}(t) \phi_{n}(x)
$$

problem (8) can be reformulated as

$$
\text { Search } C \in C^{1}\left([0, T], C^{N+1}\right) \text { such that }
$$




$$
\begin{gathered}
i \frac{d C}{d t}(t)=h C(t)+\lambda F(C(t)), \\
C(0)=C_{0},
\end{gathered}
$$

where $C_{0}$ are the coefficients of $\psi_{0}$ and $h$ the matrix of $H_{0}$ in the basis $\left(\phi_{0}, \ldots, \phi_{N}\right)$

$$
\left[C_{0}\right]_{n}=\left(\psi_{0}, \phi_{n}\right)_{L^{2}}, \quad h_{n m}=\left(H_{0} \phi_{m}, \phi_{n}\right),
$$

and where the function $F$ is defined by

$$
F(C)_{n}=\sum_{k, l, m=0}^{N} I_{k l m n} c_{k}^{*} c_{l} c_{m}
$$

with

$$
I_{k l m n}=\int_{\mathbb{R}} \phi_{k}^{*} \phi_{l} \phi_{m} \phi_{n}^{*}
$$

The efficiency of a direct implementation [26,27] of the Galerkin method described above is very poor: the calculation of the integrals $I_{k l m n}$ (which can be precomputed if the basis is small enough that the integrals can be stored in memory) scales as $O\left(N^{4} N_{p}\right)$, where $N_{p}$ is the number of grid points of the quadrature method, and the computation cost for one evaluation of the function $F$ scales as $N^{4}$ [for each of the $N$ coefficients, $O\left(N^{3}\right)$ operations are needed].

Our aim is to show that the Galerkin method becomes very efficient if $\left(\phi_{0}, \ldots, \phi_{N}\right)$ are the $N+1$ lowest eigenmodes of the harmonic oscillator $H_{0}$. In this case, indeed, the vector $F(C)$ can be computed exactly (up to round-off errors) in $O\left(N^{2}\right)$ operations. Let us recall that the eigenmodes $\left(\phi_{n}\right)_{n \in \mathbb{N}}$ of $H_{0}$ read

$$
\phi_{n}(x)=\mathcal{H}_{n}(x) e^{-x^{2} / 2},
$$

where $\mathcal{H}_{n}(x)$ is the $n$th Hermite polynomial [28], and that they satisfy

$$
H_{0} \phi_{n}=E_{n} \phi_{n} \quad \text { with } \quad E_{n}=n+\frac{1}{2} .
$$

In such a basis, the matrix $h$ is therefore diagonal: $h$ $=\operatorname{diag}\left(E_{0}, \ldots, E_{n}\right)$. In addition, for any $C \in \mathbb{C}^{N+1}$, one has

$$
F(C)_{n}=\int_{\mathbb{R}}|\psi(x)|^{2} \psi(x) \phi_{n}(x) d x,
$$

where $\psi(x)=\sum_{n=0}^{N} c_{n} \phi_{n}(x)$. The key point is now that for any $n \leqslant N$ the integrand in Eq. (11) is of the form $Q(x) e^{-2 x^{2}}$, where $Q(x)$ is a polynomial of degree lower or equal to $4 N$; each of the $N+1$ integrals can therefore be computed exactly with a Gauss-Hermite quadrature formula involving $2 N$ Gauss points [29]. More precisely, we have, for any polynomial $Q$ of degree lower or equal to $4 N$,

$$
\int_{-\infty}^{+\infty} Q(x) e^{-x^{2}} d x=\sum_{k=1}^{2 N+1} w_{k} Q\left(x_{k}\right),
$$

where $\left\{x_{k}\right\}$ are the roots of the Hermite polynomial $\mathcal{H}_{2 N+1}$ and where $\left\{w_{k}\right\}$ are convenient weights [30]. By a change of variable in integral (11), it follows that

$$
F(C)_{n}=\sum_{k=1}^{2 N+1}\left(\frac{w_{k} e^{x_{k}^{2}}}{\sqrt{2}}\right)\left|\psi\left(x_{k} / \sqrt{2}\right)\right|^{2} \psi\left(x_{k} / \sqrt{2}\right) \phi_{n}\left(x_{k} / \sqrt{2}\right) .
$$

Spectral-Galerkin methods are usually not very efficient [31]; but they can be in the specific case of the NLSE, we are interested in because of the special form of the nonlinearity.

Let us now denote by $P \in \mathcal{M}(N+1,2 N+1)$ the matrix collecting the values of the basis functions $\left(\phi_{n}\right)_{0 \leqslant n \leqslant N}$ at the Gauss points $\left(x_{k}\right)_{1 \leqslant k \leqslant 2 N+1}$ :

$$
P_{n k}=\phi_{n}\left(x_{k} / \sqrt{2}\right) \text {, }
$$

and by $\tilde{w}_{k}=w_{k} e^{x_{k}^{2}} / \sqrt{2}$. An efficient algorithm for the computation of $F(C)$ for a given $C \in \mathbb{C}^{N+1}$ reads the following.

(1) Compute the vector $\Psi \in \mathbb{C}^{2 N+1}$ defined by

$$
\Psi=P^{T} \cdot C .
$$

(2) Compute the vector $\Xi \in \mathrm{C}^{2 N+1}$ coefficient by coefficient along formula

$$
\Xi_{k}=\tilde{w}_{k}\left|\Psi_{k}\right|^{2} \Psi_{k}
$$

(3) Compute

$$
F(C)=P \cdot \Xi
$$

The vectors $C$ and $\Psi$ are the representation of the wave function $\psi$ in the spectral basis $\left\{\phi_{n}\right\}_{0 \leqslant n \leqslant N}$ and in real space (at the $2 N+1$ Gauss points $\left\{x_{k} / \sqrt{2}\right\}$ ), respectively. Steps (1) and (3) of the above algorithm scale quadratically in $N$ (these are matrix-vector products), and step (2) scales linearly in $N$. We therefore end up with an algorithmic complexity in $O\left(N^{2}\right)$.

In practice, the function $C \mapsto F(C)$ is called one or several times at each time step; of course, the matrix $P$ as well as the weights $\tilde{w}_{k}$ can be precomputed once and for all and stored in memory.

\section{B. The spectral-Galerkin method in 3D}

Let us now turn to the 3D setting and consider the rescaled equation

$$
\begin{aligned}
i \frac{\partial \psi}{\partial t}(t, x, y, z)= & {\left[\frac{\omega_{x}}{\omega_{z}} H_{0}(x)+\frac{\omega_{y}}{\omega_{z}} H_{0}(y)+H_{0}(z)\right] \psi(t, x, y, z) } \\
& +\lambda|\psi(t, x, y, z)|^{2} \psi(t, x, y, z),
\end{aligned}
$$

with

$$
\begin{gathered}
H_{0}(x)=-\frac{1}{2} \frac{\partial^{2}}{\partial x^{2}}+\frac{1}{2} x^{2}, \quad H_{0}(y)=-\frac{1}{2} \frac{\partial^{2}}{\partial y^{2}}+\frac{1}{2} y^{2}, \\
H_{0}(z)=-\frac{1}{2} \frac{\partial^{2}}{\partial z^{2}}+\frac{1}{2} z^{2} .
\end{gathered}
$$


For $\lambda \geqslant 0$, a global-in-time existence and uniqueness result is available for Eq. (12) with initial condition $\psi(t=0)=\psi_{0}$ and

$$
\begin{gathered}
\psi_{0} \in \mathcal{X}=\left\{\chi \in L^{2}\left(\mathbb{R}^{3}\right), \quad \nabla \chi \in\left[L^{2}\left(\mathbb{R}^{3}\right)\right]^{3},\right. \\
\left.\left(x^{2}+y^{2}+z^{2}\right)^{1 / 2} \chi \in L^{2}\left(\mathbb{R}^{3}\right)\right\} .
\end{gathered}
$$

On the other hand, it is well known that finite-time blow up may be observed for $\lambda<0$ and for some initial conditions [25]. As stated above, we focus here on the case where $\lambda$ $\geqslant 0$.

Following the same lines as in the Sec. II A, the approximated wave function $\psi_{N}(t)$ is expended on the spectral tensor basis set

$$
\left(\phi_{n_{x}}(x) \phi_{n_{y}}(y) \phi_{n_{z}}(z)\right)_{0 \leqslant n_{x} \leqslant N_{x}, 0 \leqslant n_{y} \leqslant N_{y}, 0 \leqslant n_{z} \leqslant N_{z}} \text {. }
$$

One therefore has

$$
\begin{aligned}
& \psi_{N}(t, x, y, z) \\
& =\sum_{n_{x}=0}^{N_{x}} \sum_{n_{y}=0}^{N_{y}} \sum_{n_{z}=0}^{N_{z}} c_{n_{x} n_{y} n_{z}}(t) \phi_{n_{x}}(x) \phi_{n_{y}}(y) \phi_{n_{z}}(z) .
\end{aligned}
$$

The equation satisfied by the three index tensor $C$ $=\left[c_{n_{x} n_{y} n_{z}}\right]$ in the Galerkin approximation formally has the same expression as in $1 \mathrm{D}$,

$$
i \frac{d C}{d t}(t)=h C(t)+\lambda F(C(t))
$$

the linear operator $h$ now being defined by

$$
[h C]_{n_{x} n_{y} n_{z}}=E_{n_{x} n_{y} n_{z}} c_{n_{x} n_{y} n_{z}}
$$

with

$$
E_{n_{x} n_{y} n_{z}}=\frac{\omega_{x}}{\omega_{z}}\left(n_{x}+\frac{1}{2}\right)+\frac{\omega_{y}}{\omega_{z}}\left(n_{y}+\frac{1}{2}\right)+\left(n_{z}+\frac{1}{2}\right),
$$

and the nonlinear function $F(C)$ by

$$
\begin{aligned}
F(C)]_{n_{x} n_{y} n_{z}}= & \int_{\mathbb{R}^{3}}|\psi(x, y, z)|^{2} \psi(x, y, z) \phi_{n_{x}}(x) \phi_{n_{y}}(y) \\
& \times \phi_{n_{z}}(z) d x d y d z,
\end{aligned}
$$

where $\psi(x, y, z)$ is given by Eq. (13).

Let us denote by $\left\{x_{k}\right\}_{1 \leqslant k \leqslant 2 N_{x}+1}, \quad\left\{y_{k}\right\}_{1 \leqslant k \leqslant 2 N_{y}+1}$, $\left\{z_{k}\right\}_{1 \leqslant k \leqslant 2 N_{z}+1}$ the roots of the Hermite polynomials $\mathcal{H}_{2 N_{x}+1}, \quad \mathcal{H}_{2 N_{y}+1}, \quad \mathcal{H}_{2 N_{z}+1} \quad$ and $\quad\left\{w_{k}^{x}\right\}_{1 \leqslant k \leqslant 2 N_{x}+1}$, $\left\{w_{k}^{y}\right\}_{1 \leqslant k \leqslant 2 N_{y}+1},\left\{w_{k}^{z}\right\}_{1 \leqslant k \leqslant 2 N_{z}+1}$ the associated summation weights. Let us also introduce the matrices $P_{x} \in \mathcal{M}\left(N_{x}\right.$ $\left.+1,2 N_{x}+1\right), P_{y} \in \mathcal{M}\left(N_{y}+1,2 N_{y}+1\right), P_{z} \in \mathcal{M}\left(N_{z}+1,2 N_{z}\right.$ +1 ) defined by

$$
\begin{gathered}
{\left[P_{x}\right]_{n_{x} k_{x}}=\phi_{n_{x}}\left(x_{k_{x}} / \sqrt{2}\right), \quad\left[P_{y}\right]_{n_{y} k_{y}}=\phi_{n_{y}}\left(y_{k_{y}} / \sqrt{2}\right),} \\
{\left[P_{z}\right]_{n_{z} k_{z}}=\phi_{n_{z}}\left(z_{k_{z}} / \sqrt{2}\right),}
\end{gathered}
$$

and the weights

$$
\tilde{w}_{k_{x}}^{x}=\frac{w_{k_{x}}^{x} e^{x_{k_{x}}^{2}}}{\sqrt{2}}, \quad \tilde{w}_{k_{z}}^{y}=\frac{w_{k_{y}}^{y} e^{y_{k_{y}}^{2}}}{\sqrt{2}}, \quad \tilde{w}_{k_{z}}^{z}=\frac{w_{k_{z}}^{z} e^{z_{k_{z}}^{2}}}{\sqrt{2}} .
$$

The following algorithm for the computation of $F(C)$ scales in $O\left(N N_{x} N_{y} N_{z}\right)$ where $N=\max \left(N_{x}, N_{y}, N_{z}\right)$.

(1) Set $\Psi^{S S S}=C$.

(2) Compute $\Psi_{n_{x} n_{y} k_{z}}^{S S R}=\sum_{n_{z}=0}^{N_{z}}\left[P_{z}\right]_{n_{z} k_{z}} \Psi_{n_{x} n_{y} n_{z}}^{S S S}$,

$$
O\left(N_{x} N_{y} N_{z}^{2}\right) \text { operations. }
$$

(3) Compute $\Psi_{n_{x} k_{y} k_{z}}^{S R R}=\sum_{n_{y}=0}^{N_{y}}\left[P_{y}\right]_{n_{y} k_{y}} \Psi_{n_{x} n_{y} k_{z}}^{S S R}$,

$$
O\left(N_{x} N_{y}^{2} N_{z}\right) \text { operations. }
$$

(4) Compute $\Psi_{k_{x} k_{y} k_{z}}^{R R R}=\sum_{n_{x}=0}^{N_{x}}\left[P_{x}\right]_{n_{x} k_{x}} \Psi_{n_{x} k_{y} k_{z}}^{S R R}$,

$$
O\left(N_{x}^{2} N_{y} N_{z}\right) \text { operations. }
$$

(5) Compute $\Xi_{k_{x} k_{y} k_{z}}^{R R R}=\tilde{w}_{k_{x}}^{x} \tilde{w}_{k_{y}}^{y} \tilde{w}_{k_{z}}^{z}\left|\Psi_{k_{x} k_{y} k_{z}}^{R R R}\right|^{2} \Psi_{k_{x} k_{y} k_{z}}^{R R R}$,

$$
O\left(N_{x} N_{y} N_{z}\right) \text { operations. }
$$

(6) Compute $\Xi_{k_{x} k_{y} n_{z}}^{R R S}=\sum_{k_{z}=1}^{2 N_{z}+1}\left[P_{z}\right]_{n_{z} k_{z}} \Xi_{k_{x} k_{y} k_{z}}^{R R R}$,

$O\left(N_{x} N_{y} N_{z}^{2}\right)$ operations.

(7) Compute $\Xi_{k_{x} n_{y} n_{z}}^{R S S}=\sum_{k_{y}=1}^{2 N_{y}+1}\left[P_{y}\right]_{n_{y} k_{y}} \Xi_{k_{x} k_{y} n_{z}}^{R R S}$,

$$
O\left(N_{x} N_{y}^{2} N_{z}\right) \text { operations. }
$$

(8) Compute $\Xi_{n_{x} n_{y} n_{z}}^{S S S}=\sum_{k_{x}=1}^{2 N_{x}+1}\left[P_{x}\right]_{n_{x} k_{x}} \Xi_{k_{x} n_{y} n_{z}}^{R S S}$,

$$
O\left(N_{x}^{2} N_{y} N_{z}\right) \text { operations. }
$$

(9) $\operatorname{Set} F(C)=\Xi^{S S S}$.

In the above formulation, the superscripts $S$ and $R$ stand for spectral and real space representations, respectively. In other words, steps (2)-(4) constitute the successive transformation of the wave function from the spectral basis to a spatial representation on the series of points of the Gauss-Hermite 
quadrature. The nonlinear term of the Hamiltonian is then calculated in this spatial representation [step (5)], while steps (6) -(8) correspond to the inverse transform back to the spectral basis. It is this procedure of forward and backward transformation that allows us to obtain a much better scaling than the implementation of Eq. (10).

The scaling of the above algorithm $\left[O\left(N^{4}\right)\right.$ if $N_{x}=N_{y}$ $=N_{z}$ ] has to be compared with the scaling of fast Fourier transform based algorithms which scale in $O\left(N_{p}^{3} \log _{2}\left(N_{p}\right)\right)$, where $N_{p}$ is the number of grid points per direction. The main interest of the spectral method is that for a similar accuracy, the number of spectral basis functions per direction (here denoted by $N$ ) can usually be chosen much smaller than the number $N_{p}$ of grid points per direction. This is especially true when the problem considered displays a symmetry in one or more of the directions, in which case the basis set used in the Galerkin approximation Eq. (13) can be restricted to even harmonic-oscillator functions (in the corresponding direction). We will come back on this important feature of the spectral method in Sec. IV.

\section{Exploiting spherical or cylindrical symmetry}

When $\omega_{x}=\omega_{y}=\omega_{z}$ the one-particle Hamiltonian possesses spherical symmetry. If the initial condition $\psi_{0}=\psi(t$ $=0)$ has the same symmetry, then the wave function $\psi(t)$ is spherical symmetric for any $t>0: \quad \psi(t, x, y, z)=\psi(t, r)$, where $r=\left(x^{2}+y^{2}+z^{2}\right)^{1 / 2}$ is the radial coordinate. Equation (4) leads to the effective 1D dynamics

$$
i \frac{\partial \psi}{\partial t}=\left[-\frac{1}{2 r^{2}} \frac{\partial}{\partial r}\left(r^{2} \frac{\partial}{\partial r}\right)+\frac{r^{2}}{2}+\lambda|\psi|^{2}\right] \psi .
$$

Let us now define the function

$$
\chi(t, r)= \begin{cases}\sqrt{2 \pi} r \psi(t, r) & \text { if } r>0 \\ -\sqrt{2 \pi} r \psi(t,-r) & \text { if } r<0 .\end{cases}
$$

It is easy to check that $\chi$ actually satisfies the 1D NLSE

$$
i \frac{\partial \chi}{\partial t}=H_{0} \chi+\lambda \frac{|\chi|^{2}}{2 \pi r^{2}} \chi
$$

Besides, for any $t>0$ the function $\chi(t): r \mapsto \chi(t, r)$ is odd and belongs to $L^{2}(\mathbb{R})$ since

$$
\int_{-\infty}^{+\infty}|\chi(t, r)|^{2} d r=\int_{0}^{+\infty} 4 \pi r^{2}|\psi(t, r)|^{2} d r=1 .
$$

It can thus be expanded on the odd modes of the harmonic oscillator:

$$
\chi(t, r)=\sum_{n=0}^{+\infty} c_{n}(t) \phi_{2 n+1}(r) .
$$

A spectral-Galerkin approximation can now be used. The vector $C(t) \in \mathrm{C}^{N+1}$ collecting the coefficients $\left(c_{k}(t)\right)_{0 \leqslant k \leqslant N}$ of the approximated wave function

$$
\chi_{N}(t, r)=\sum_{n=0}^{N} c_{n}(t) \phi_{2 n+1}(r)
$$

obeys once again a dynamics of the form

$$
i \frac{d C}{d t}(t)=h C(t)+\lambda F(C(t))
$$

Here

$$
h=\operatorname{diag}\left(E_{2 n+1}\right) \quad \text { with } \quad E_{2 n+1}=2 n+\frac{3}{2}
$$

and

$$
[F(C)]_{n}=\int_{\mathbb{R}} \frac{|\chi(r)|^{2}}{2 \pi r^{2}} \chi(r) \phi_{2 n+1}(r) d r
$$

where $\chi(r)=\sum_{n=0}^{N} c_{n} \phi_{2 n+1}(r)$. As for any $0 \leqslant n \leqslant N$, $\phi_{2 n+1}(r)=r P_{2 n}(r) e^{-r^{2} / 2}$ where $P_{2 n}$ is a polynomial of degree equal to $2 n$, it follows that the above integrals can be computed exactly with $4 N$ Gauss points.

Let us now turn to the cylindrical symmetry when (for instance) $\omega_{x}=\omega_{y}$ and when the initial data reads $\psi_{0}(x, y, z)$ $=\psi_{0}(r, z)$ with $r=\left(x^{2}+y^{2}\right)^{1 / 2}$. In this case, the cylindrical symmetry is preserved by the dynamics so that for any $t$ $>0, \quad \psi(t, x, y, z)=\psi(t, r, z)$ and the time evolution of $\psi(t, r, z)$ is then governed by the $2 \mathrm{D}$ equation

$$
\begin{aligned}
i \frac{\partial \psi}{\partial t}= & {\left[\frac{\omega_{x}}{\omega_{z}}\left\{-\frac{1}{2 r} \frac{\partial}{\partial r}\left(r \frac{\partial}{\partial r}\right)+\frac{r^{2}}{2}\right\}+\left(-\frac{1}{2} \frac{\partial^{2}}{\partial z^{2}}+\frac{z^{2}}{2}\right)\right.} \\
& \left.+\lambda|\psi|^{2}\right] \psi,
\end{aligned}
$$

set on the spatial domain $\mathbb{R}^{+} \times \mathbb{R}$. Defining a new function $\chi(t, r, z)$ on the space domain $\mathbb{R}^{2}$ by

$$
\chi(t, r, z)= \begin{cases}\psi(t, r, z) & \text { if } r>0 \\ \psi(t,-r, z) & \text { if } r<0,\end{cases}
$$

it occurs that $\chi$ satisfies

$$
\begin{aligned}
i \frac{\partial \chi}{\partial t}= & {\left[\frac{\omega_{x}}{\omega_{z}}\left(-\frac{1}{2} \frac{\partial^{2}}{\partial r^{2}}+\frac{r^{2}}{2}\right)+\left(-\frac{1}{2} \frac{\partial^{2}}{\partial z^{2}}+\frac{z^{2}}{2}\right)-\frac{\omega_{x}}{\omega_{z}} \frac{1}{2 r} \frac{\partial}{\partial r}\right.} \\
& \left.+\lambda|\chi|^{2}\right] \chi
\end{aligned}
$$

on the space domain $\mathbb{R}^{2}$, and that, by construction, the function $r \mapsto \chi(t, r, z)$ is even. A spectral-Galerkin approximation is obtained by expanding the wave function on the spectral tensor basis set

$$
\left(\phi_{2 n_{r}}(r) \phi_{n_{z}}(z)\right)_{0 \leqslant n_{r} \leqslant N_{r}, 0 \leqslant n_{z} \leqslant N_{z}} .
$$


The coefficients $\left(c_{n_{r} n_{z}}\right)_{0 \leqslant n_{r} \leqslant N_{r}, 0 \leqslant n_{z} \leqslant N_{z}}$ of the expansion are solution of an equation of the same form as above,

$$
i \frac{d C}{d t}(t)=h C(t)+\lambda F(C(t)) .
$$

The main difference is that in this case, the linear map $h$ takes into account the operator $-(1 / 2 r)(\partial / \partial r)$ :

$$
\begin{aligned}
{[h C]_{n_{r} n_{z}}=} & {\left[\frac{\omega_{x}}{\omega_{z}}\left(n_{r}+\frac{1}{2}\right)+\left(n_{z}+\frac{1}{2}\right)\right] C_{n_{r} n_{z}} } \\
& -\frac{1}{2} \frac{\omega_{x}}{\omega_{z}} \sum_{m_{r}=0}^{N_{r}}\left(\frac{1}{r} \frac{d \phi_{2 m_{r}}}{d r}, \phi_{2 n_{r}}\right){ }_{L^{2}} C_{m_{r} n_{z}} .
\end{aligned}
$$

Let us remark that the scalar product $\left((1 / r)\left(d \phi_{2 m_{r}} / d r\right), \phi_{n_{r}}\right)_{L^{2}}$ is well defined since the first derivative of $\phi_{2 n_{r}}$ is of the form $r P_{2 n_{r}}(r) e^{-r^{2} / 2}$ where $P_{2 n_{r}}$ is a polynomial of degree $2 n_{r}$; in addition, it can be computed exactly by numerical integration with $2 n_{r}$ Gauss points. It is worth pointing out that the "Hamiltonian" in Eq. (16) is not self-adjoint because of the term $-(1 / 2 r)(\partial / \partial r)$ and that the $L^{2}$ norm of $\chi(t)$ is not a conserved quantity; on the other hand, the $L^{2}$ norm of $\chi(t)$ for the measure $r d r d z$ is conserved.

\section{TIME DISCRETIZATION}

When a spectral-Galerkin method is used to discretize the space variables, one ends up with a finite-dimensional dynamical system of the form

$$
i \frac{d C}{d t}(t)=h C(t)+\lambda F(C(t)),
$$

with initial condition $C(t=0)=C_{0}$. We then use a basic fourth-order Runge-Kutta method [32] to solve Eq. (17). Let us mention that, as the Hamiltonian character of the NLSE is preserved by the spectral-Galerkin discretization, it would be possible to resort to symplectic methods [33]; such algorithms, which are particularly advised for long time evolution, are, however, not tested in the present work.

We will also use a grid method, based on the splitoperator method, to serve as a benchmark for the spectral algorithm we have just detailed. We recall below the main features of this approach.

The wave function at time $\tau+\Delta \tau$ can be obtained from the wave function at $\tau$ according to

$$
\psi(\tau+\Delta \tau)=\hat{U}(\tau, \tau+\Delta \tau) \psi(\tau),
$$

with the propagator $\hat{U}(\tau, \tau+\Delta \tau)$ being expressed, for sufficiently small intervals $\Delta \tau$, as

$$
\hat{U}(\tau, \tau+\Delta \tau)=\exp [-i H(\tau) \Delta \tau],
$$

where $H(\tau)$ is the Hamiltonian of Eq. (4). As the potential and nonlinear components of the Gross-Pitaevskii Hamiltonian do not commute with the kinetic operator, we apply the split-operator method [34] to obtain

$$
\begin{aligned}
\exp [-i H(\tau) \Delta \tau]= & \exp \left[-i T \frac{\Delta \tau}{2}\right] \exp \left[-i\left(V+\lambda|\psi|^{2}\right) \Delta \tau\right] \\
& \times \exp \left[-i T \frac{\Delta \tau}{2}\right]+O\left(\Delta \tau^{3}\right)
\end{aligned}
$$

with $T$ the kinetic operator and $V$ the trapping potential. The middle term is diagonal in position space, while the kinetic part is diagonal in momentum space. A fast Fourier transform is thus used before application of the kinetic operator, followed by the inverse transform. Note that if the intermediate wave function at time $\tau+\Delta \tau$ is not needed, the two successive kinetic operators half steps can be combined. From a previous study [35], it appears that the split-operator method is the fastest algorithm for solving a NLSE on a grid.

\section{RESULTS}

The first test we perform is the propagation of the ground stationary state (obtained from the time-independent GPE solved by a method based on the optimal damping algorithm [36-38]), while monitoring the value of the coefficients $c(\tau)$ of the expansion (13). For the spherically symmetric case, we require that the relative error on the $c_{0}$ coefficient (which has the largest absolute value) be inferior to $10^{-8}$, i.e., $\left.|| c_{0}(\tau)\right|^{2}-\left.\left|c_{0}(\tau=0)\right|^{2}|/| c_{0}(\tau=0)\right|^{2} \leqslant 10^{-8} \forall \tau$ $\in[0,100]$. This criterion also results in an absolute error of all coefficients $\left|c_{n}(\tau)\right|^{2}-\left|c_{n}(\tau=0)\right|^{2} \leqslant 10^{-8}$. We have also checked that the phase of the coefficients is correct, by calculating $\left|c_{n}(\tau)-c_{n}(\tau=0) e^{-i \mu \tau}\right|^{2} /\left|c_{n}(\tau)\right|^{2}$, where $\mu$ is the chemical potential of the ground stationary state of the GPE [6], and this value indeed is less than $10^{-12}$.

In this 1D case, we need $N=20$ basis functions for $\lambda$ $=100$, and the resulting time step for the Runge-Kutta propagator is $\Delta \tau=0.005$. If $\lambda=1000$, the basis set used should be slightly larger, $N=26$, with a smaller time step $\Delta \tau=0.0025$ to insure that the above error criteria are met. The resulting propagation time up to $\tau=100$ is $8.9 \mathrm{~s}$ for $\lambda$ $=100$ (calculated on an Athlon $1.2 \mathrm{GHz}$ processor running under Linux, using the NAG Fortran 95 compiler at the $-\mathrm{O} 2$ level of optimization) and $28.3 \mathrm{~s}$ for $\lambda=1000$. If we double the size of the basis set, we get a CPU time of $32.9 \mathrm{~s}$ for $\lambda$ $=100$, showing the expected $O\left(N^{2}\right)$ scaling of the algorithm in $1 \mathrm{D}$.

Comparing now with the grid method described in Sec. III, we use $N_{p}=64$ grid points in the range $-8 \leqslant r \leqslant 8$. The time step used is $\Delta \tau=0.00025$, resulting in a propagation time of $10.3 \mathrm{~s}$, which is slightly longer than what we obtain using the Runge-Kutta method.

We now apply our algorithm to study the dynamics of trapped condensates. Referring again to the spherically symmetric case, we start with the stationary ground state for an isotropic trap frequency $\omega$. We then let this initial state $\psi_{0}$ evolve in a trap of frequency $\omega / 2$, as illustrated in Fig. 1, corresponding to an experiment where the frequency of the potential trapping the condensate would be instantaneously reduced by a factor of 2 . The corresponding time-evolving wave function $|\psi(t, r)|^{2}$ is shown in Fig. 2, for $\lambda=10$. We must note that the values of $\lambda$ we give correspond to the 


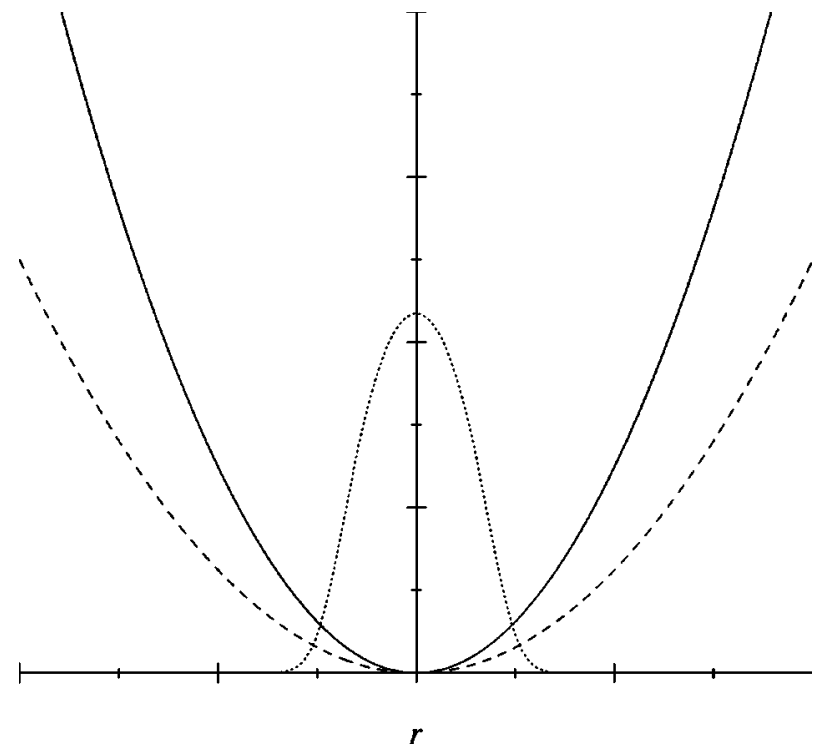

FIG. 1. Trapping potentials $\omega$ (solid line) and $\omega / 2$ (dashed line) used to simulate the breathing modes of a condensate. The wave function $|\psi(r)|^{2}$ of the stationary state for potential $\omega$ with $\lambda$ $=100$ is also given (dotted line).

condensate in the initial $\omega$-frequency trap, the effective value being used for the time evolution is thus scaled by $1 / \sqrt{2}$ [see Eq. (5)], while $\tau$ is rescaled with respect to the final trap frequency $\omega / 2$. We can see the "breathing" of the condensate as it expands and recontracts in the trap.

It is also interesting to look at the effect of the value of the nonlinear parameter on the breathing frequency of the condensate, as seen in Fig. 3. First, we note that the initial density at the center of the trap is lower for bigger values of $\lambda$, which is expected because of the corresponding higher inter-

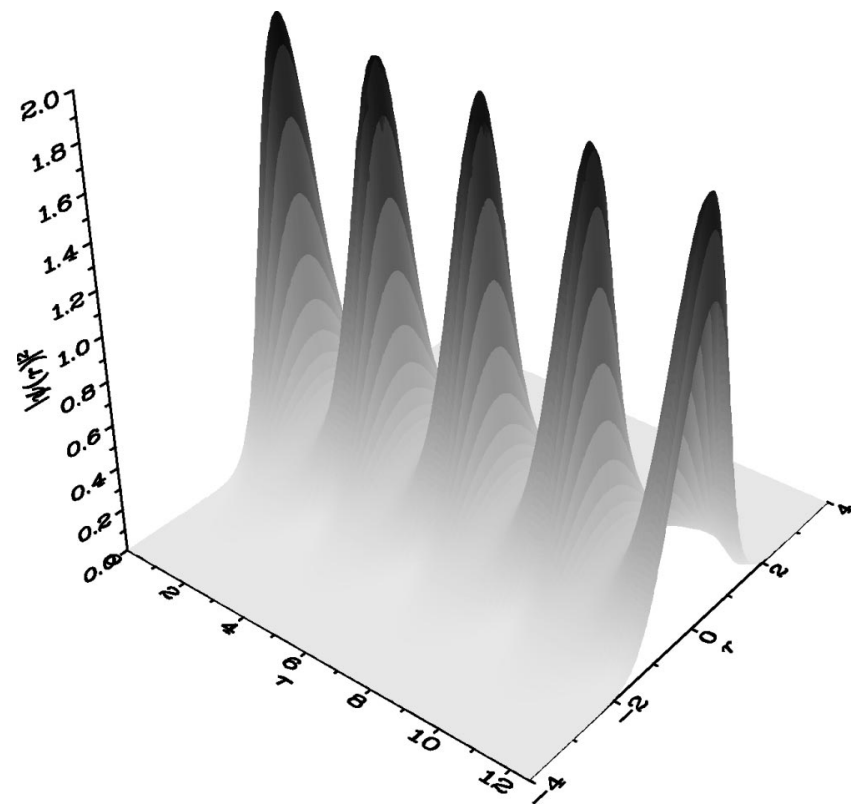

FIG. 2. "Breathing" of the condensate after expansion from a trap of frequency $\omega$ to $\omega / 2$. The density profile $|\psi(r)|^{2}$ is given as a function of time $\tau$ (scaled with respect to the final trap frequency $\omega / 2$ ) for an initial $\lambda=10$ ground stationary state.

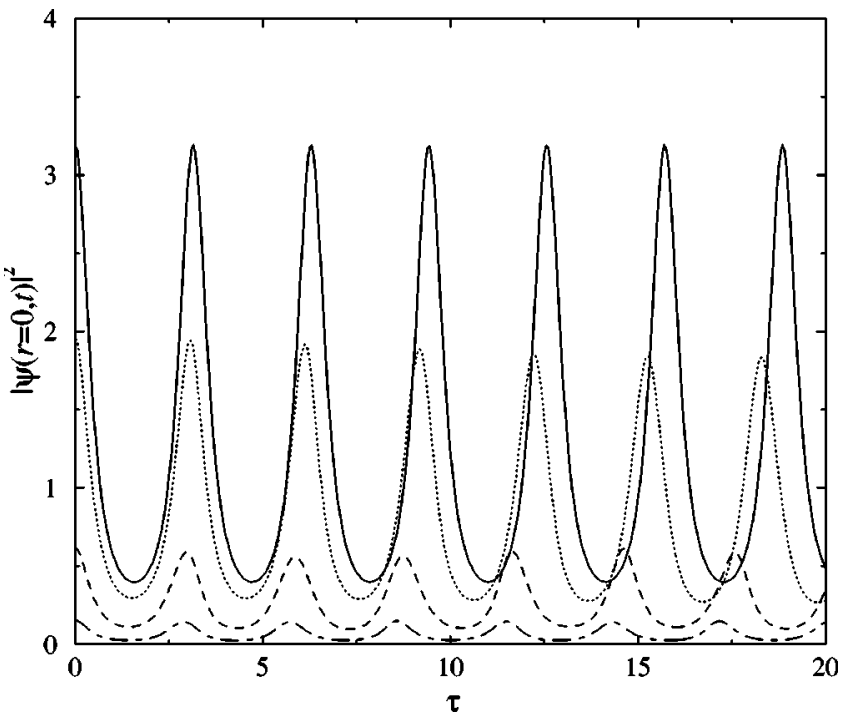

FIG. 3. "Breathing" of the condensate after expansion from a trap of frequency $\omega$ to $\omega / 2$. The value of the wave function in the center of the trap, $|\psi(r=0)|^{2}$, is given as a function of time $\tau$ (scaled with respect to the final trap frequency $\omega / 2$ ) for $\lambda$ equal to 0 (solid line), 10 (dotted line), 100 (dashed line), and 1000 (dotdashed line).

particle repulsion. Starting from an unperturbed harmonic oscillator $(\lambda=0)$, for which the complete cycle time is $\tau$ $=4 \pi$ with recurrences every $\tau=\pi$, we observe that the oscillation frequency of the condensate in the trap increases with a greater value of $\lambda$.

For the 3D case, we will study the scissor mode $[39,40]$ of a trapped condensate. We consider a pancake-shaped condensate, formed in an anisotropic trap with $\omega_{x}=\omega_{y} \ll \omega_{z}$, see Fig. 4. The $y$ and $z$ axes of the trap are instantaneously rotated, at $t=0$, by an angle $\theta$ around the $x$ axis. The condensate then starts to oscillate in the trap, leading to the socalled scissor mode.

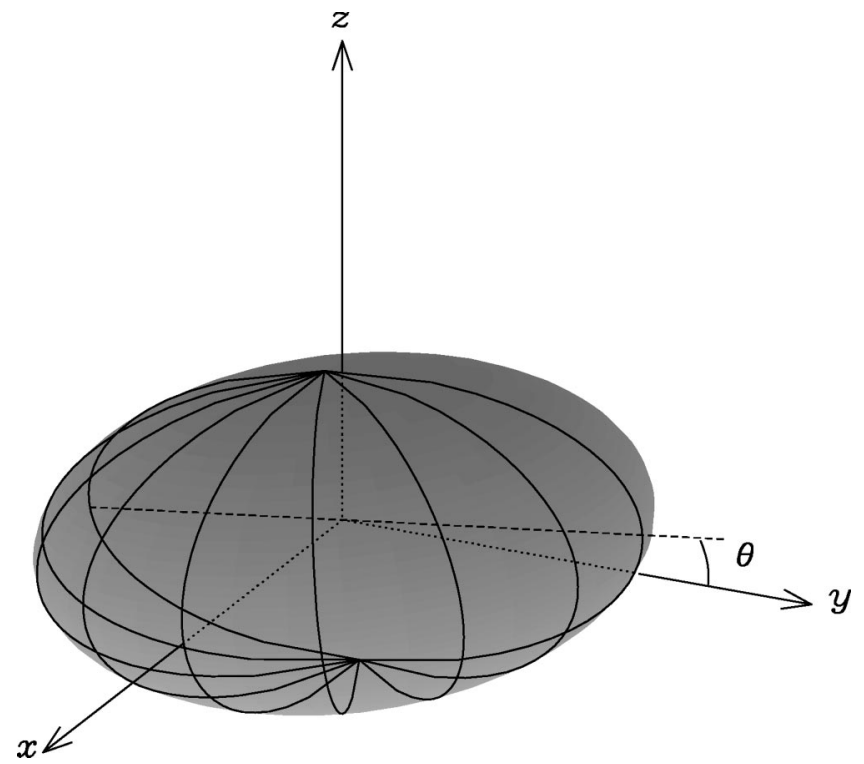

FIG. 4. Representation of the study a condensate's scissor mode. The condensate is initially tilted with respect to the trap's $y$ and $z$ axes by an angle $\theta$. 
Using the parameters of the experiment of Maragò et al. [40], we first determine the stationary state for a condensate of $N=10^{4}{ }^{87} \mathrm{Rb}$ atoms in a trap with $\omega_{z}=255 \mathrm{~Hz}, \omega_{x} / \omega_{z}$ $=\omega_{y} / \omega_{z}=1 / \sqrt{8}$, resulting in a value $\lambda=147.1$. The condensate is then tilted by an angle of $\theta=3.6^{\circ}$, with the trapping frequency $\omega_{z}$ reduced by $2 \%$, resulting in a new value of $\lambda$ $=148.6$. We then calculate the free evolution of this tilted condensate. We report, in Fig. 5, the angle between the condensate (as determined by the main inertia axis) and the $y$ axis, as a function of time for the free evolution of the condensate in a trap. The oscillation frequency, in these conditions, is found to be 1.105 (in rescaled units), corresponding to $276 \mathrm{~Hz}$. This simulation was done using a basis set of $N$ $=29$ functions in each dimension, using a time step $\Delta \tau$ $=0.005$. The calculation time for a propagation of duration $\tau$ is then $\approx 1735 \mathrm{~s}$. The main advantage of using a spectralGalerkin method, as noted in Sec. II B, is that in this case we can restrict the basis set in the $x$ dimension by using only even harmonic-oscillator functions, since the reflection symmetry with respect to the $y O z$ plane is conserved. The number of functions is thus reduced to $N_{x}=15$, resulting in a decrease of CPU time to $\approx 1030 \mathrm{~s}$ for $1 \tau$. This compares favorably with the grid method, for which an equivalent calculation with $64 \times 64 \times 64$ grid points takes $\approx 1700 \mathrm{~s}$ (using the same time step and grid spacing as for the $1 \mathrm{D}$ grid method).

\section{CONCLUSION}

We have presented the application of a spectral-Galerkin method to the numerical solution of the Gross-Pitaevskii equation, describing a Bose-Einstein condensate (BEC) trapped in a harmonic potential well. This method is based on the decomposition of the condensate wave function on the a basis set of eigenmodes of the harmonic oscillator, while the nonlinear term in the GPE is calculated using the GaussHermite quadrature. The resulting algorithm scales in $O\left(N^{4}\right)$ for a full 3D problem (where $N$ is the number of basis functions used per direction), which is slightly worse than the $O\left(N_{p}^{3} \log _{2} N_{p}\right)$ scaling obtained for grid-based Fourier methods. Nevertheless, the required number of basis functions needed for a given problem can be much smaller than the number of grid points $N_{p}$, allowing for fast and efficient calculations using the spectral method. We have shown how the propagation in time can be carried out using a RungeKutta method on a set of coupled ordinary differential equations.

This method is akin to the DVR approach [21,22], which relies on the fact that matrix elements of the nonlinear term can be approximately evaluated to sufficiently high accuracy using an $N$-point rule based Gauss quadrature. Our approach has the advantage that, for the basis chosen, there are no

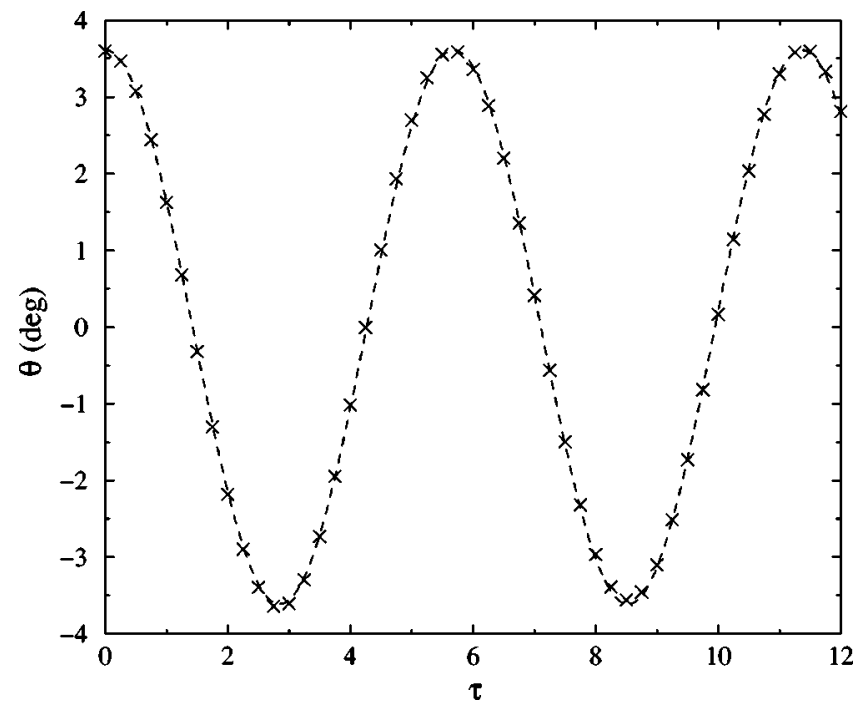

FIG. 5. Time evolution of the angle $\theta$ for the scissor mode. The crosses correspond to the angle resulting from the time-dependent calculation, along with the corresponding fit $\theta=3.6 \cos (1.105 \tau)$ (dashed line).

approximations in the computation of these integrals. Let us, however, remark that the same property can hold within the DVR method by a suitable choice of weighted polynomials. The main distinction between the usual DVR approach and our method is that we treat the kinetic and potential terms of the Hamiltonian conjointly, as detailed in Sec. II.

We have successfully applied our algorithm to simulate two different dynamical aspects of trapped BECs. Making use of the spherical symmetry of an isotropic trapping potential, we used an effective 1D equation to study the breathing of a condensate that is allowed to expand from more confining trap to a looser one. In the 3D case, we have looked at the scissor modes of a pancake-shaped condensate, for which the trapping potential is suddenly rotated along one axis.

Future work will focus on the implementation of better time-evolution algorithms on our spectral method and on its possible parallelization. Extensions will also be made to consider other terms in the Gross-Pitaevskii Hamiltonian, such as the potential created by the interaction with a laser field, or coupled Gross-Pitaevskii equations used in the simulation of two-species condensates [41] or of the formation of molecules in atomic condensates $[42,43]$.

\section{ACKNOWLEDGMENTS}

We thank L. DiMenza, O. Dulieu, J. Mary, F. MasnouSeeuws, and P. Pellegrini for stimulating discussions. Part of this work was carried out using the computer resources of IDRIS (Orsay, France).
[1] M.H. Anderson, J.R. Ensher, M.R. Matthews, C.E. Wieman, and E.A. Cornell, Science 269, 198 (1995).

[2] C.C. Bradley, C.A. Sackett, J.J. Tollett, and R.G. Hulet, Phys. Rev. Lett. 75, 1687 (1995).
[3] K.B. Davis, M.-O. Mewes, M.R. Andrews, N.J. van Druten, D.S. Durfee, D.M. Kurn, and W. Ketterle, Phys. Rev. Lett. 75, 3969 (1995).

[4] E.P. Gross, Nuovo Cimento 20, 454 (1961). 
[5] L.P. Pitaevskii, Zh. Eksp. Teor. 40, 646 (1961) [Sov. Phys. JETP 13, 451 (1961)].

[6] F. Dalfovo, S. Giorgini, L.P. Pitaevskii, and S. Stringari, Rev. Mod. Phys. 71, 463 (1999).

[7] A. Röhrl, M. Naraschewski, A. Schenzle, and H. Wallis, Phys. Rev. Lett. 78, 4143 (1997).

[8] K. Bongs, S. Burger, G. Birkl, K. Sengstock, W. Ertmer, K. Rzążewski, A. Sanpera, and M. Lewenstein, Phys. Rev. Lett. 83, 3577 (1999).

[9] M.R. Matthews, B.P. Anderson, P.C. Haljan, D.S. Hall, M.J. Holland, J.E. Williams, C.E. Wieman, and E.A. Cornell, Phys. Rev. Lett. 83, 3358 (1999).

[10] M. Modugno, F. Dalfovo, C. Fort, P. Maddaloni, and F. Minardi, Phys. Rev. A 62, 063607 (2000).

[11] J.-P. Martikainen, K.-A. Suominen, L. Santos, T. Schulte, and A. Sanpera, Phys. Rev. A 64, 063602 (2001).

[12] W. Hoston and L. You, Phys. Rev. A 53, 4254 (1996).

[13] R.J. Ballagh, K. Burnett, and T.F. Scott, Phys. Rev. Lett. 78, 1607 (1997).

[14] P.D. Drummond and K.V. Kheruntsyan, Phys. Rev. A 63, 013605 (2000).

[15] P.A. Ruprecht, M.J. Holland, K. Burnett, and M. Edwards, Phys. Rev. A 51, 4704 (1995).

[16] S.K. Adhikari, Phys. Rev. E 62, 2937 (2000).

[17] B. Jackson, J.F. McCann, and C.S. Adams, J. Phys. B 31, 4489 (1998).

[18] R. Baer, Phys. Rev. A 62, 063810 (2000).

[19] J.J. Tollett, C.C. Bradley, C.A. Sackett, and R.G. Hulet, Phys. Rev. A 51, R22 (1995).

[20] W. Petrich, M.H. Anderson, J.R. Ensher, and E.A. Cornell, Phys. Rev. Lett. 74, 3352 (1995).

[21] B.I. Schneider and D.L. Feder, Phys. Rev. A 59, 2232 (1999).

[22] D.L. Feder, M.S. Pindzola, L.A. Collins, B.I. Schneider, and C.W. Clark, Phys. Rev. A 62, 053606 (2000).

[23] F. Pereira Dos Santos, J. Léonard, J. Wang, C.J. Barrelet, F. Perales, E. Rasel, C.S. Unnikrishnan, M. Leduc, and C. CohenTannoudji, Phys. Rev. Lett. 86, 3459 (2001).

[24] J.L. Roberts, N.R. Claussen, J.P. Burke, Jr., C.H. Greene, E.A. Cornell, and C.E. Wieman, Phys. Rev. Lett. 81, 5109 (1998).

[25] T. Cazenave, An Introduction to Nonlinear Schrödinger Equa- tions, 3rd ed., Textos de Métodos Matemáticos Vol. 26 (Instituto de Matemática-UFRJ, Rio de Janeiro, 1996).

[26] M. Edwards, R.J. Dodd, C.W. Clark, and K. Burnett, J. Res. Natl. Inst. Stand. Technol. 101, 553 (1996).

[27] M. Edwards, R.J. Dodd, C.W. Clark, P.A. Ruprecht, and K. Burnett, Phys. Rev. A 53, R1950 (1996).

[28] C. Cohen-Tannoudji, B. Diu, and F. Laloë, Quantum Mechanics (Wiley, New York, 1992).

[29] A.H. Stroud and D. Secrest, Gaussian Quadrature Formulas (Prentice-Hall, Englewood Cliffs, NJ, 1966).

[30] P.J. Davis and I. Polonsky, in Handbook of Mathematical Functions, edited by M. Abramowitz and I.A. Stegun (Dover, New York, 1965), Chap. 25, pp. 875-924.

[31] Y. Maday and C. Bernardi, in Handbook of Numerical Analysis, edited by P. Ciarlet and J.-L. Lions (Elsevier, Amsterdam, 2000), Vol. 5, pp. 209-486.

[32] W.H. Press, S.A. Teukolsky, W.T. Vetterling, and B.P. Flannery, Numerical Recipes in FORTRAN, 2nd ed. (Cambridge University Press, Cambridge, 1992).

[33] Y.-F. Tang, L. Vázquez, F. Zhang, and V.M. Pérez-García, Comput. Math. Appl. 32, 73 (1996).

[34] M.D. Feit, J.A. Fleck, Jr., and A. Steiger, J. Comput. Phys. 47, 412 (1982).

[35] T.R. Taha and M.J. Ablowitz, J. Comput. Phys. 55, 203 (1984).

[36] E. Cancès and C. Le Bris, Int. J. Quantum Chem. 79, 82 (2000).

[37] E. Cancès, in Mathematical Models and Methods for Ab Initio Quantum Chemistry, edited by M. Defranceschi and C. Le Bris, Lecture Notes in Chemistry Vol. 74 (Springer, Berlin, 2000), pp. 17-43.

[38] E. Cancès and C.M. Dion (unpublished).

[39] D. Guéry-Odelin and S. Stringari, Phys. Rev. Lett. 83, 4452 (1999).

[40] O.M. Maragò, S.A. Hopkins, J. Arlt, E. Hodby, G. Hechenblaikner, and C.J. Foot, Phys. Rev. Lett. 84, 2056 (2000).

[41] M. Trippenbach, K. Góral, K. Rzążzewski, B. Malomed, and Y.B. Band, J. Phys. B 33, 4017 (2000).

[42] E. Timmermans, P. Tommasini, R. Côté, M. Hussein, and A. Kerman, Phys. Rev. Lett. 83, 2691 (1999).

[43] D.J. Heinzen, R. Wynar, P.D. Drummond, and K.V. Kheruntsyan, Phys. Rev. Lett. 84, 5029 (2000). 